\title{
17. The Correlation Between the Movements of the Cardia and the Autonomic Nervous Center
}

\author{
Hiroshi Sato, Takashi Tanaka, Shuichi Mizuno, Tokuo Kazuno, \\ Tokuo KaZawa, Osamu Inami, Setsuo Takeuchi \\ III Department of Surgery, Nihon University School of Medicine \\ Tokuzo KoJrma and Naomasa Ito \\ II Department of Anatomy, Nihon University School of Medicine
}

Using the Horsley-Clarke stereotaxic instruments the changes of the cardia movements in the cat were observed caused by the electrical stimulation of the hypothalamus, midbrain and oblongata and destruction of the hypothalamus.

The movements and EMG of the cardia were recorded by means of the balloon method and concentric needle electrodes.

1. The cardia movements were caused by the stimulation of the following points of the brain.

a. In the rostral and middle portions of the hypothalamus the points were found scattered moderately as a whole, while in the transitional level between the hypothalamus and the midbrain only a few points were found dorsally.

b. Considerable numbers of the points were found at dorsal vagal nucleus and adjacent portion in the medulla oblongata.

2. In 4 of 34 cats pathological dilation of the esophagus like idiopathic were made by the electrical destruction of the hypothalamus especially nucl. hypothalami ventromedialis and areas closed to this nucleus. The results of the dilation of esophagus were ascertained by $\mathrm{X}$-ray operation and recording of endo-pressure of cardia by electrical manometer. In addition, by the histological examination the degenerations of the myentric nerve plexus were revealed and clinically the symptoms of dysphagia, vomiting and diarrhea etc. were found.

\section{On the Relation Between Muscle Tonus and Reciprocal Innervation \\ NAKAAKIRA TSUKAHARA \\ Department of Neurophysiology, Institute of Brain Research, University of Tokyo \\ Hirotaro NARABayashI \\ Section of Neurology, Juntendo University}

It has been presumed that hyperactivity of the gamma system may cause the 